\title{
ALGEBRO-GEOMETRIC SEMISTABILITY OF POLARIZED TORIC MANIFOLDS*
}

\author{
HAJIME $\mathrm{ONO}^{\dagger}$
}

\begin{abstract}
Let $\Delta \subset \mathbb{R}^{n}$ be an $n$-dimensional integral Delzant polytope. It is well-known that there exist the $n$-dimensional compact toric manifold $X_{\Delta}$ and a very ample $\left(\mathbb{C}^{\times}\right)^{n}$-equivariant line bundle $L_{\Delta}$ on $X_{\Delta}$ associated with $\Delta$. In the present paper, we give a necessary and sufficient condition for Chow semistability of $\left(X_{\Delta}, L_{\Delta}^{i}\right)$ for a maximal torus action. We then see that asymptotic (relative) Chow semistability implies (relative) K-semistability for toric degenerations, which is proved by Ross and Thomas [10].
\end{abstract}

Key words. Chow stability, K-stability, polarized toric manifold.

AMS subject classifications. 14L24, 14M25, 52B20.

1. Introduction. Let $X$ be a compact complex manifold and $L$ an ample line bundle on $X$. We call the pair $(X, L)$ a polarized manifold. One of the main subjects in Kähler geometry is the existence problem of Kähler metrics with constant scalar curvature, and more generally, of extremal Kähler metrics. It is now conjectured that the existence of such metrics in $c_{1}(L)$ is equivalent to some algebro-geometric stability of $(X, L)$. One of the difficulty to consider this problem is the existence of many notions of stability. We have to specify the exact one. Though a lot of progress has been made recently in this problem by Tian, Donaldson and many other researchers, we do not know what is appropriate stability yet. Therefore it is important to know relations among various notions of stability.

In the present paper we investigate the following semistabilities of polarized toric manifolds, asymptotic Chow semistability, K-semistability and relative Ksemistability.

Let $\Delta \subset \mathbb{R}^{n}$ be an $n$-dimensional integral Delzant polytope. Namely, $\Delta$ satisfies the following conditions (in [7] polytopes satisfying these conditions are called absolutely simple) :

1. All the vertices $\mathbf{w}_{1}, \ldots, \mathbf{w}_{d}$ of $\Delta$ are contained in $\mathbb{Z}^{n}$.

2. For each $l$, there are just $n$ rational edges $e_{l, 1}, \ldots, e_{l, n}$ of $\Delta$ emanating from $\mathbf{w}_{l}$.

3. The primitive vectors with respect to the edges $e_{l, 1}, \ldots, e_{l, n}$ generate the lattice $\mathbb{Z}^{n}$ over $\mathbb{Z}$.

It is well-known that $n$-dimensional integral Delzant polytopes correspond to $n$ dimensional compact toric manifolds with $\left(\mathbb{C}^{\times}\right)^{n}$-equivariant very ample line bundles. The reader is referred to [7] for example.

Let $\Delta \subset \mathbb{R}^{n}$ be an $n$-dimensional integral Delzant polytope. We denote the Ehrhart polynomial of $\Delta$ by $E_{\Delta}(t)$, which is a polynomial of degree $n$ satisfying

$$
E_{\Delta}(i)=\#\left(i \Delta \cap \mathbb{Z}^{n}\right)=\#\left(\Delta \cap(\mathbb{Z} / i)^{n}\right)
$$

for each positive integer $i$. In Section 3 we will consider representations of the complex torus $\left(\mathbb{C}^{\times}\right)^{E_{\Delta}(i)}$. The character group of this torus can be identified with $\left\{i \Delta \cap \mathbb{Z}^{n} \rightarrow\right.$

\footnotetext{
*Received September 1, 2011; accepted for publication June 25, 2012.

$\dagger$ Department of Mathematics, Faculty of Science, Saitama University, Shimo-Okubo 255, Sakuraku Saitama-shi, 338-8570 Japan (hono@rimath.saitama-u.ac.jp).
} 
$\mathbb{Z}\} \simeq\left\{\Delta \cap(\mathbb{Z} / i)^{n} \rightarrow \mathbb{Z}\right\} \simeq \mathbb{Z}^{E_{\Delta}(i)}$. Then we denote

$$
\begin{gathered}
W(i \Delta)=\left\{i \Delta \cap \mathbb{Z}^{n} \rightarrow \mathbb{R}\right\} \simeq\left\{\Delta \cap(\mathbb{Z} / i)^{n} \rightarrow \mathbb{R}\right\} \simeq \mathbb{R}^{E_{\Delta}(i)}, \\
W(i \Delta)_{\mathbb{Q}}=\left\{i \Delta \cap \mathbb{Z}^{n} \rightarrow \mathbb{Q}\right\} \simeq\left\{\Delta \cap(\mathbb{Z} / i)^{n} \rightarrow \mathbb{Q}\right\} \simeq \mathbb{Q}^{E_{\Delta}(i)} .
\end{gathered}
$$

We identify $W(i \Delta)$ with its dual space by the scalar product

$$
(\varphi, \psi)=\sum_{\mathbf{a} \in \Delta \cap(\mathbb{Z} / i)^{n}} \varphi(\mathbf{a}) \psi(\mathbf{a})
$$

For each $\varphi \in W(i \Delta)$, define a concave piecewise linear function $g_{\varphi}: \Delta \rightarrow \mathbb{R}$ as follows. Let

$$
G_{\varphi}=\text { the convex hull of } \bigcup_{\mathbf{a} \in \Delta \cap(\mathbb{Z} / i)^{n}}\{(\mathbf{a}, t) \mid t \leq \varphi(\mathbf{a})\} \subset \mathbb{R}^{n} \times \mathbb{R} .
$$

Then we define $g_{\varphi}$ by

$$
g_{\varphi}(\mathbf{x})=\max \left\{t \mid(\mathbf{x}, t) \in G_{\varphi}\right\}, \quad \mathbf{x} \in \Delta
$$

and denote

$$
P L(\Delta ; i)=\left\{g_{\varphi} \mid \varphi \in W(i \Delta)\right\}, \quad P L_{\mathbb{Q}}(\Delta ; i)=\left\{g_{\varphi} \mid \varphi \in W_{\mathbb{Q}}(i \Delta)\right\}
$$

The following is the main theorem of this paper.

ThEOREM 1.1. Let $\Delta \subset \mathbb{R}^{n}$ be an $n$-dimensional integral Delzant polytope. For a positive integer $i$, the Chow form of $\left(X_{\Delta}, L_{\Delta}^{i}\right)$ is $T_{i \Delta}^{\mathbb{C}}$-semistable if and only if

$$
P_{\Delta}(i ; g)=E_{\Delta}(i) \int_{\Delta} g d v-\operatorname{Vol}(\Delta) \sum_{\mathbf{a} \in \Delta \cap(\mathbb{Z} / i)^{n}} g(\mathbf{a}) \geq 0
$$

holds for any $g \in P L(\Delta ; i)$. Here $T_{i \Delta}^{\mathbb{C}}$ is the subtorus of $\left(\mathbb{C}^{\times}\right)^{E_{\Delta}(i)}$ defined by

$$
T_{i \Delta}^{\mathbb{C}}=\left\{\left(t_{1}, \ldots, t_{E_{\Delta}(i)}\right) \in\left(\mathbb{C}^{\times}\right)^{E_{\Delta}(i)} \mid \prod_{j=1}^{E_{\Delta}(i)} t_{j}=1\right\}
$$

This paper is organized as follows. In Section 2 we review some basics of geometric invariant theory briefly for later use. In Section 3, we first define the Chow form of submanifolds of a projective space. Then Chow semistability is defined as GIT-semistability of the Chow form. Finally we prove Theorem 1.1. In Section 4, we compare asymptotic Chow semistability with K-semistability of polarized toric manifolds through Theorem 1.1. In Section 5, by analogy with Chow semistability, we define the notion of relative Chow semistability of polarized toric manifolds in toric sense and prove that asymptotic relative Chow semistability in toric sense implies relative K-semistability for toric degenerations.

Acknowledgements. The author would like to thank the referee for valuable comments. 
2. GIT-stability. Let $G$ be a reductive Lie group. Suppose that $G$ acts on a complex vector space $V$ linearly. We call a nonzero vector $v \in V G$-semistable if the closure of the orbit $G v$ does not contain the origin. Similarly we call $p \in \mathbb{P}(V)$ $G$-semistable if any representative of $p$ in $V \backslash\{\mathbf{0}\}$ is $G$-semistable. It is well-known that there is the following useful criterion for $v$ being $G$-semistable, see [6].

Proposition 2.1 (Hilbert-Mumford criterion, [6]). $p \in \mathbb{P}(V)$ is $G$-semistable if and only if $p$ is $H$-semistable for each maximal torus $H \subset G$.

Hence it is important to study $G$-semistability when $G$ is isomorphic to an algebraic torus $\left(\mathbb{C}^{\times}\right)^{n}$. Let $G$ be isomorphic to $\left(\mathbb{C}^{\times}\right)^{n}$. Then a $G$-module $V$ is decomposed as

$$
V=\sum_{\chi \in \chi(G)} V_{\chi}, \quad V_{\chi}=\{v \in V \mid t \cdot v=\chi(t) v, \forall t \in G\},
$$

where $\chi(G) \simeq \mathbb{Z}^{n}$ is the character group of the torus $G$.

Definition 2.2. Let $v=\sum_{\chi \in \chi(G)} v_{\chi}$ be a nonzero vector in $V$. The weight polytope $\mathrm{Wt}_{G}(v) \subset \chi(G) \otimes_{\mathbb{Z}} \mathbb{R}$ of $v$ is the convex hull of $\left\{\chi \in \chi(G) \mid v_{\chi} \neq \mathbf{0}\right\}$ in $\chi(G) \otimes_{\mathbb{Z}} \mathbb{R}$.

The following fact about $G$-semistability is standard.

Proposition 2.3. Let $G$ be isomorphic to $\left(\mathbb{C}^{\times}\right)^{n}$. Suppose that $G$ acts on a complex vector space $V$ linearly. Then a nonzero vector $v \in V$ is $G$-semistable if and only if the weight polytope $\mathrm{Wt}_{G}(v)$ contains the origin.

Let $G=\left(\mathbb{C}^{\times}\right)^{n}$ and $H$ be the subtorus

$$
H=\left\{\left(t_{1}, \ldots, t_{n-1},\left(t_{1} \cdots t_{n-1}\right)^{-1}\right) \mid\left(t_{1}, \ldots, t_{n-1}\right) \in\left(\mathbb{C}^{\times}\right)^{n-1}\right\} \simeq\left(\mathbb{C}^{\times}\right)^{n-1} .
$$

Then the weight polytope $\mathrm{Wt}_{H}(v) \subset \chi(H) \otimes_{\mathbb{Z}} \mathbb{R} \simeq \mathbb{R}^{n-1}$ equals to $\pi\left(\mathrm{Wt}_{G}(v)\right)$, where the linear map $\pi: \mathbb{R}^{n} \rightarrow \mathbb{R}^{n-1}$ is given as $\left(x_{1}, \ldots, x_{n-1}, x_{n}\right) \mapsto\left(x_{1}-x_{n}, \ldots, x_{n-1}-\right.$ $\left.x_{n}\right)$.

Therefore we see the following.

Proposition 2.4. $v$ is $H$-semistable if and only if there exists $t \in \mathbb{R}$ such that $(t, \ldots, t) \in \mathrm{Wt}_{G}(v)$.

3. Chow semistability of polarized toric manifolds. We first define the Chow form of irreducible projective varieties. See [3] for more detail.

Definition 3.1. Let $X \subset \mathbb{C} P^{N}$ be an $n$-dimensional irreducible subvariety of degree $d$. It is easy to see that the subset $Z_{X}$ of the Grassmannian $\operatorname{Gr}\left(N-n-1, \mathbb{C} P^{N}\right)$ defined by

$$
Z_{X}=\left\{L \in \operatorname{Gr}\left(N-n-1, \mathbb{C} P^{N}\right) \mid L \cap X \neq \emptyset\right\}
$$

is an irreducible hypersurface of degree $d$. Hence $Z_{X}$ is given by the vanishing of a degree $d$ element $\left[R_{X}\right] \in \mathbb{P}\left(\mathcal{B}_{d}\left(N-n-1, \mathbb{C} P^{N}\right)\right)$, where $\mathcal{B}\left(N-n-1, \mathbb{C} P^{N}\right)=$ $\oplus_{d} \mathcal{B}_{d}\left(N-n-1, \mathbb{C} P^{N}\right)$ is the graded coordinate ring of the Grassmannian. We call $R_{X}$ the Chow form of $X$.

Since the special linear group $S L(N+1, \mathbb{C})$ acts naturally on $\mathcal{B}_{d}\left(N-n-1, \mathbb{C} P^{N}\right)$, we can consider the $S L(N+1, \mathbb{C})$-stability of the Chow form $R_{X}$. 
Definition 3.2. Let $X \subset \mathbb{C} P^{N}$ be an $n$-dimensional irreducible subvariety of degree $d$. We call $X$ Chow semistable if the Chow form $R_{X}$ is $S L(N+1, \mathbb{C})$-semistable. When $X$ is not Chow semistable $X$ is called Chow unstable.

Definition 3.3. Let $L$ be a very ample line bundle on a complex manifold $X$. $(X, L)$ is called Chow semistable when $\Psi(X) \subset \mathbb{P}\left(H^{0}(X ; L)^{*}\right)$ is Chow semistable. Here $\Psi: X \rightarrow \mathbb{P}\left(H^{0}(X ; L)^{*}\right)$ is the Kodaira embedding.

Definition 3.4. Let $(X, L)$ be a polarized manifold. We call $(X, L)$ asymptotically Chow semistable if $\left(X, L^{i}\right)$ is Chow semistable for any sufficiently large integer $i$.

Now we investigate Chow semistability of $\left(X_{\Delta}, L_{\Delta}^{i}\right)$ for an $n$-dimensional integral Delzant polytope $\Delta$ and a positive integer $i$. By Hilbert-Mumford criterion, Proposition 2.1, $H$-semistability of the Chow form is essential for any maximal torus $H$ of $S L\left(E_{\Delta}(i), \mathbb{C}\right)$. As a specific case, we take the following maximal torus;

$$
T_{i \Delta}^{\mathbb{C}}=\left(\mathbb{C}^{\times}\right)^{E_{\Delta}(i)} \cap S L\left(E_{\Delta}(i), \mathbb{C}\right) .
$$

Here $\left(\mathbb{C}^{\times}\right)^{E_{\Delta}(i)} \subset G L\left(E_{\Delta}(i), \mathbb{C}\right)$ is the torus consisting of diagonal matrices. Note that $T_{i \Delta}^{\mathbb{C}}$ is the subtorus of $\left(\mathbb{C}^{\times}\right)^{E_{\Delta}(i)}$ given as $(2.2)$.

Proposition 3.5 ([3], see also [8]). Let $C h_{i \Delta}$ be the weight polytope of the Chow form of $\left(X_{\Delta}, L_{\Delta}^{i}\right)$ for $\left(\mathbb{C}^{\times}\right)^{E_{\Delta}(i)}$-action. Then the affine hull of $C h_{i \Delta}$ in $W(i \Delta)$ is $(3.1)$

$$
\left\{\varphi \in W(i \Delta) \mid \sum_{\mathbf{a} \in \Delta \cap(\mathbb{Z} / i)^{n}} \varphi(\mathbf{a})=(n+1) ! \operatorname{Vol}(i \Delta), \sum_{\mathbf{a} \in \Delta \cap(\mathbb{Z} / i)^{n}} i \varphi(\mathbf{a}) \mathbf{a}=(n+1) ! \int_{i \Delta} \mathbf{x} d v\right\},
$$

where $\mathbf{x}$ is the position vector.

Therefore we have the following by Proposition 2.4.

Proposition 3.6. Let $\Delta \subset \mathbb{R}^{n}$ be an $n$-dimensional integral Delzant polytope. Then the Chow form of $\left(X_{\Delta}, L_{\Delta}^{i}\right)$ is $T_{i \Delta}^{\mathbb{C}}$-semistable if and only if

$$
\frac{i^{n}(n+1) ! \operatorname{Vol}(\Delta)}{E_{\Delta}(i)} d_{i \Delta} \in C h_{i \Delta},
$$

where $d_{i \Delta} \in W(i \Delta)$ is

$$
d_{i \Delta}(\mathbf{a})=1, \quad \mathbf{a} \in \Delta \cap(\mathbb{Z} / i)^{n}
$$

Proof of Theorem 1.1. The condition (3.2) is equivalent to the following condition.

$$
\forall \varphi \in W(i \Delta), \max \left\{(\varphi, \psi) \mid \psi \in C h_{i \Delta}\right\} \geq \frac{(n+1) ! \operatorname{Vol}(i \Delta)}{E_{\Delta}(i)}\left(\varphi, d_{i \Delta}\right)
$$

The left hand side of (3.3) is a constant multiple of the integral of $g_{\varphi}$.

Lemma 3.7 (Lemma 1.9 of [3] Chapter 7). For any $\varphi \in W(i \Delta)$,

$$
\max \left\{(\varphi, \psi) \mid \psi \in C h_{i \Delta}\right\}=i^{n}(n+1) ! \int_{\Delta} g_{\varphi} d v
$$


holds.

By this lemma, (3.3) is equivalent to

$$
\forall \varphi \in W(i \Delta), \int_{\Delta} g_{\varphi} d v \geq \frac{\operatorname{Vol}(\Delta)}{E_{\Delta}(i)} \sum_{\mathbf{a} \in \Delta \cap(\mathbb{Z} / i)^{n}} g_{\varphi}(\mathbf{a}) .
$$

Applying (1.1) to linear functions, we have the following corollary.

Corollary $3.8([8])$. Let $\Delta \subset \mathbb{R}^{n}$ be an n-dimensional integral Delzant polytope. If $\left(X_{\Delta}, L_{\Delta}^{i}\right)$ is Chow semistable for a positive integer $i$ then we have

$$
\sum_{\mathbf{a} \in \Delta \cap(\mathbb{Z} / i)^{n}} \mathbf{a}=\frac{E_{\Delta}(i)}{\operatorname{Vol}(\Delta)} \int_{\Delta} \mathbf{x} d v
$$

4. K-semistability. For polarized manifolds, the notion of K-stability was introduced by Donaldson in [1]. In toric case, restricting test configurations to toric degenerations, he also defined the following notion.

Definition $4.1([1])$. Let $\left(X_{\Delta}, L_{\Delta}\right)$ be an n-dimensional polarized toric manifold. If the inequality

$$
\frac{\operatorname{Vol}(\partial \Delta)}{\operatorname{Vol}(\Delta)} \int_{\Delta} h d v-\int_{\partial \Delta} h d \sigma \leq 0
$$

holds for any rational convex piecewise linear function $h: \Delta \rightarrow \mathbb{R},\left(X_{\Delta}, L_{\Delta}\right)$ is called K-semistable for toric degenerations.

Ross and Thomas, in [10], showed that asymptotically Chow semistable polarized manifold is K-semistable. In toric case, we can partly prove this fact by Theorem 1.1.

Proposition 4.2. If $\left(X_{\Delta}, L_{\Delta}\right)$ is asymptotically Chow semistable, then $\left(X_{\Delta}, L_{\Delta}\right)$ is $K$-semistable for toric degenerations.

Proof. Let $h: \Delta \rightarrow \mathbb{R}$ be a rational convex piecewise linear function. Then $g=-h$ is a rational concave piecewise linear function on $\Delta$. Thus there is a positive integer $i$ such that $g \in P L_{\mathbb{Q}}(\Delta ; i)$. Note here that if $g \in P L(\Delta ; i)$ (resp. $g \in P L_{\mathbb{Q}}(\Delta ; i)$ ), then $g \in P L(\Delta ; k i)$ (resp. $g \in P L_{\mathbb{Q}}(\Delta ; k i)$ ) for any positive integer $k$. Since $\left(X_{\Delta}, L_{\Delta}\right)$ is asymptotically Chow semistable,

$$
P_{\Delta}(k i ; g)=E_{\Delta}(k i) \int_{\Delta} g d v-\operatorname{Vol}(\Delta) \sum_{\mathbf{a} \in \Delta \cap(\mathbb{Z} /(k i))^{n}} g(\mathbf{a}) \geq 0
$$

holds for any positive integer $k$ by Theorem 1.1.

It is well-known that the coefficients of $n$-th and $(n-1)$-th order terms of the Ehrhart polynomial of $\Delta$ are $\operatorname{Vol}(\Delta)$ and $\operatorname{Vol}(\partial \Delta) / 2$ respectively;

$$
E_{\Delta}(t)=\operatorname{Vol}(\Delta) t^{n}+\frac{\operatorname{Vol}(\partial \Delta)}{2} t^{n-1}+O\left(t^{n-2}\right) .
$$

Here the volume form $d \sigma$ on $\partial \Delta$ is defined as follows. On a facet $\left\{h_{r}=c_{r}\right\} \cap \Delta$ of $\Delta$, where $h_{r}$ is a primitive linear form, $d h_{r} \wedge d \sigma$ equals to the Euclidean volume form $d v$. 
Hence we have

$$
E_{\Delta}(k i)=\operatorname{Vol}(\Delta)(k i)^{n}+\frac{\operatorname{Vol}(\partial \Delta)}{2}(k i)^{n-1}+O\left((k i)^{n-2}\right) .
$$

Moreover by Lemma 3.3 of [12],

$$
\sum_{\mathbf{a} \in \Delta \cap(\mathbb{Z} / k i)^{n}} g(\mathbf{a})=(k i)^{n} \int_{\Delta} g d v+\frac{(k i)^{n-1}}{2} \int_{\partial \Delta} g d \sigma+O\left((k i)^{n-2}\right)
$$

holds. Therefore we have

$$
P_{\Delta}(k i ; g)=\frac{\operatorname{Vol}(\Delta)}{2}\left(\frac{\operatorname{Vol}(\partial \Delta)}{\operatorname{Vol}(\Delta)} \int_{\Delta} g d v-\int_{\partial \Delta} g d \sigma\right)(k i)^{n-1}+O\left((k i)^{n-2}\right) .
$$

This proposition follows immediately from (4.2) and (4.6).

By (4.6), the following proposition trivially holds.

Proposition 4.3. An $n$-dimensional polarized toric manifold $\left(X_{\Delta}, L_{\Delta}\right)$ is $K$ semistable for toric degenerations if and only if one of the following holds for any positive integer $i$ and $g \in P L_{\mathbb{Q}}(\Delta, i)$ :

$$
P_{\Delta}(k i ; g)>0, \quad \forall k \gg 0 \quad \text { or } \quad \frac{P_{\Delta}(k i ; g)}{(k i)^{n-1}} \rightarrow 0 \quad(k \rightarrow \infty) .
$$

By Theorem 1.1, asymptotic Chow semistablity of $\left(X_{\Delta}, L_{\Delta}\right)$ corresponds to nonnegativity of the polynomial $P_{\Delta}(k i ; g)$. On the other hand, $\left(X_{\Delta}, L_{\Delta}\right)$ is K-semistable for toric degenerations if and only if the leading coefficients of the polynomial $P_{\Delta}(k i ; g)$ is non-negative. Hence asymptotic Chow semistability is much stronger than Ksemistability. In fact we showed in [9] that there exists a 7-dimensional K-polystable toric Fano manifold which is not asymptotically Chow semistable. In this case, by Corollary 1.7 of [8], there is a rational linear function $l$ on $\Delta$ such that $P_{\Delta}(i ; l)<0$ for any sufficiently large integer $i$. Hence $l$ is a destabilizing object for asymptotic Chow semistability. On the other hand, since the Futaki invariant of this Fano manifold vanishes, we see that the leading coefficient of $P_{\Delta}(i ; l)$

$$
\frac{\operatorname{Vol}(\partial \Delta)}{\operatorname{Vol}(\Delta)} \int_{\Delta} l d v-\int_{\partial \Delta} l d \sigma
$$

vanishes. Thus

$$
\frac{P_{\Delta}(i ; l)}{i^{n-1}} \rightarrow 0 \quad(i \rightarrow \infty)
$$

Therefore $l$ is not a destabilizing object for K-semistability.

5. Relative K-semistability. The notion of relative K-stability was defined in [11]. As Definition 4.1, we define the following.

DeFINITION $5.1([12])$. Let $\left(X_{\Delta}, L_{\Delta}\right)$ be an $n$-dimensional polarized toric manifold. If the inequality

$$
\int_{\Delta}\left(\frac{\operatorname{Vol}(\partial \Delta)}{\operatorname{Vol}(\Delta)}+\theta_{\Delta}\right) h d v-\int_{\partial \Delta} h d \sigma \leq 0
$$


holds for any rational convex piecewise linear function $h: \Delta \rightarrow \mathbb{R},\left(X_{\Delta}, L_{\Delta}\right)$ is called relatively $K$-semistable for toric degenerations. Here $\theta_{\Delta}: \Delta \rightarrow \mathbb{R}$ is the affine linear function, defined in Lemma 1.1 of [12], corresponding to the extremal vector field of $\left(X_{\Delta}, L_{\Delta}\right)$.

For any sufficiently large integer $i$ and a continuous function $g$ on $\Delta$ we have

$$
\sum_{\mathbf{a} \in \Delta \cap(\mathbb{Z} / i)^{n}} \theta_{\Delta}(\mathbf{a}) g(\mathbf{a})=i^{n} \int_{\Delta} \theta_{\Delta} g d v+O\left(i^{n-1}\right) .
$$

Thus for any $g \in P L_{\mathbb{Q}}(\Delta ; i)$ and sufficiently large integer $k$

$$
\begin{aligned}
& Q_{\Delta}(k i ; g)=E_{\Delta}(k i) \int_{\Delta} g d v+\operatorname{Vol}(\Delta) \sum_{\mathbf{a} \in \Delta \cap(\mathbb{Z} /(k i))^{n}}\left(\frac{1}{2 k i} \theta_{\Delta}(\mathbf{a})-1\right) g(\mathbf{a}) \\
& =\frac{\operatorname{Vol}(\Delta)}{2}\left\{\int_{\Delta}\left(\frac{\operatorname{Vol}(\partial \Delta)}{\operatorname{Vol}(\Delta)}+\theta_{\Delta}\right) g d v-\int_{\partial \Delta} g d \sigma\right\}(k i)^{n-1}+O\left((k i)^{n-2}\right) .
\end{aligned}
$$

Thus, as Proposition 4.3, we see the following.

Proposition 5.2. An n-dimensional polarized toric manifold $\left(X_{\Delta}, L_{\Delta}\right)$ is relatively K-semistable for toric degenerations if and only if one of the following holds for any positive integer $i$ and $g \in P L_{\mathbb{Q}}(\Delta, i)$ :

$$
Q_{\Delta}(k i ; g) \geq 0, \forall k \gg 0 \quad \text { or } \quad \frac{Q_{\Delta}(k i ; g)}{(k i)^{n-1}} \rightarrow 0 \quad(k \rightarrow \infty) .
$$

Finally we see that some GIT-semistability implies relative K-semistability for toric degenerations.

Definition 5.3. $\left(X_{\Delta}, L_{\Delta}^{i}\right)$ is relatively Chow semistable for $T_{i \Delta}^{\mathbb{C}}$-action if

$$
\frac{i^{n}(n+1) ! \operatorname{Vol}(\Delta)}{E_{\Delta}(i)}\left(d_{i \Delta}-\frac{1}{2 i} \theta_{\Delta}\right) \in C h_{i \Delta} .
$$

$\left(X_{\Delta}, L_{\Delta}\right)$ is asymptotically relatively Chow semistable in toric sense if there exists a positive integer $i_{0}$ such that for any integer $i \geq i_{0}(5.5)$ holds.

Proposition 5.4. If $\left(X_{\Delta}, L_{\Delta}\right)$ is asymptotically relatively Chow semistable in toric sense, then $\left(X_{\Delta}, L_{\Delta}\right)$ is relatively K-semistable for toric degenerations.

Proof. Suppose that $\left(X_{\Delta}, L_{\Delta}\right)$ is asymptotically relatively Chow semistable in toric sense. By Lemma 3.7, it is equivalent to

$$
\forall \varphi \in W(i \Delta), \int_{\Delta} g_{\varphi} d v \geq \frac{\operatorname{Vol}(\Delta)}{E_{\Delta}(i)} \sum_{\mathbf{a} \in \Delta \cap(\mathbb{Z} / i)^{n}}\left(1-\frac{1}{2 i} \theta_{\Delta}(\mathbf{a})\right) \varphi(\mathbf{a})
$$

for any integer $i \geq i_{0}$. Hence it implies that for any $g \in P L(\Delta ; i)$ and positive integer $k$

$$
Q_{\Delta}(k i ; g) \geq 0 .
$$

Therefore $\left(X_{\Delta}, L_{\Delta}\right)$ is relatively $\mathrm{K}$-semistable for toric degenerations by Proposition 5.2. 


\section{REFERENCES}

[1] S. K. Donaldson, Scalar curvature and stability of toric varieties, J. Differential Geom., 62 (2002), pp. 289-349.

[2] W. Fulton, Introduction to Toric Varieties, no. 131, in Annals of Mathematics Studies, Princeton University Press, Princeton, NJ, 1993.

[3] I. M. Gelfand, M. M. Kapranov, and A. V. Zelevinsky, Discriminants, Resultants and Multidimensional Determinants, Mathematics: Theory \& Applications. Birkhäuser Boston Inc., Boston, MA, 1994.

[4] M. M. Kapranov, B. Sturmfels, and A. V. Zelevinsky, Chow polytopes and general resultants, Duke Math. J., 67:1 (1992), pp. 189-218.

[5] D. Mumford, Stability of projective varieties, Enseignement Math. (2), 23:1-2 (1977), pp. 39110.

[6] D. Mumford, J. Fogarty, and F. Kirwan, Geometric Invariant Theory, Third edition, Ergebnisse der Mathematik und ihrer Grenzgebiete (2), 34, Springer-Verlag, Berlin, 1994.

[7] T. OdA, Convex bodies and algebraic geometry, Ergebnisse der Mathematik und ihrer Grenzgebiete (3), 15, Springer-Verlag, Berlin, 1988.

[8] H. Ono, A necessary condition for Chow semistability of polarized toric manifolds, J. Math. Soc. Japan, 63:4 (2011), pp. 1377-1389.

[9] H. Ono, Y. Sano, and N. Yotsutani, An example of asymptotically Chow unstable manifolds with constant scalar curvature, Ann. Inst. Fourier, 62:4 (2012), pp. 1265-1287.

[10] J. Ross And R. Thomas, A study of the Hilbert-Mumford criterion for the stability of projective varieties, J. Algebraic Geom., 10 (2007), pp. 201-255.

[11] G. SzÉKelyHidi, Extremal metrics and K-stability, Bull. Lond. Math. Soc., 39:1 (2007), pp. 7684.

[12] B. Zhou And X. Zhu, Relative K-stability and modified K-energy on toric manifolds, Adv. Math., 219 (2008), pp. 1327-1362. 